RECEIVED

Alli, 041997

OSTI

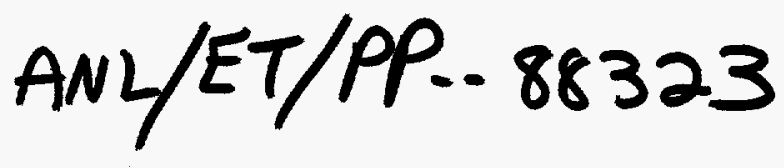

\title{
Intelligent Hand-Portable Proliferation Sensing System
}

'S. L. Dieckman, G. A. Bostrom, L. G. Waterfield, J. A. Jendrzejczyk, S. Ahuja, and A. C. Raptis

Instrumentation \& Nondestructive Evaluation Section, Energy Technology Division, Argonne National Laboratory, 9700 South Cass Avenue, Argonne II 60439-4825

The submitted manuscript has been authored by a contractor of the U.S. Government under contract No. W-31-109ENG-38. Accordingly, the U.S. Government retains a non-exclusive, royalty-free license to publish or reproduce the published form of this contribution, or allow others to do so, for U.S. Government purposes.

An invited article to be published in the Arms Control and Nonproliferation Technology Review, George Staehle Scientific Editor, Published by the U.S. Department of Energy, December 1995.

*We are pleased to acknowledge that the financial support for this work was provided by the U.S. Department of Energy, Office of Intelligence and National Security, Office of Research and Development, under Contract W31-109-Eng-38.

DISTAIBUTION OF THS DOCUMENT IS UNLIMITED 


\title{
Intelligent Hand-Portable Proliferation Sensing System
}

\author{
S. L. Dieckman, G. A. Bostrom, L. G. Waterfield, \\ J. A. Jendrzejczyk, S. Ahuja, and A. C. Raptis \\ Argonne National Laboratory \\ Argonne IL 60439
}

Argonne National Laboratory, with support from DOE's Office of Nonproliferation and National Security, is currently developing an intelligent hand-portable sensor system. This system is designed specifically to support the intelligence community with the task of in-field sensing of nuclear proliferation and related activities. Based upon pulsed laser photo-ionization time-of-flight mass spectrometry technology, this novel sensing system is capable of quickly providing a molecular or atomic analysis of specimens. The system is capable of analyzing virtually any gas phase molecule, or molecule that can be induced into the gas phase by (for example) sample heating. This system has the unique advantages of providing unprecedented portability, excellent sensitivity, tremendous fieldability, and a high performance/cost ratio. The system will be capable of operating in a highly automated manner for on-site inspections, and easily modified for other applications such as perimeter monitoring aboard a plane or drone.

A diagram of the sensor system is shown in Figure 1. The complete spectrometer including computer system is approximately notebook size ( 4 " $\left.\times 9 " \times 11^{\prime \prime}\right)$ and when combined with a scant weight of 15 to 18 pounds, provides unprecedented portability. The spectrometer's main components consists of a miniature laser, cryocooler, cold finger, vacuum pump, a leak valve, ion detector, and an electrostatic ion lens. Auxiliary components (not presented in the diagram but included in the size and weight specifications) include the instrument control and data acquisition electronics, a global positioning sensor (GPS), and the battery pack. 
Based upon photo-ionization followed by time-of-flight mass spectrometry, this technique provides simplicity in instrument function and control, the ability to analyze a wide range of molecules, high operational stability, and high sensitivity. Operation of the spectrometer system is performed by leaking gas phase molecules into the spectrometer (which is maintained at high vacuum). The molecules collide with the liquid nitrogen temperature cold finger where many freeze onto the surface. A short ultra violet laser pulse (3 nanoseconds) is then applied to the frozen molecules, causing the formation of ionized molecules near the surface. These ionized molecules (ions) are then accelerated by applying high voltages to the electrostatic ion lens. The ions are propelled away from the surface of the cold finger, fly down the flight tube, and ultimately collide with the ion detector. Because the ions are all accelerated with the same potential, low mass ions travel quickly, while higher mass ions travel more slowly. Data is then acquired as a function of time, providing a spectrum of the sample's molecular masses (commonly called a mass spectrum). Segregation and detection of the molecules by mass as performed in this system provides the ability to identify and quantify the molecules or atoms of interest.

The spectrometer system is controlled using a notebook personal computer and operated with the aid of an advanced man-machine interface. Designed to require minimal operator training, the system performs highly automated data acquisition, data processing, and instrument control with the aid of user-friendly mouse driven software. The software is being developed using a high level "Windows" based graphical programming language that provides tremendous analytical and instrumentational control capabilities while maintaining simplicity in design and programming. Using this popular hardware platform provides the means for effortless upgrading to take advantage of future increases in computer and peripheral performance. Additionally, the software development package allows easy customization to meet the needs of specific users or applications. Pattern recognition for automated analysis of data through ion fragment matching with a known database will soon be installed. Other software features will include accurate sample location using the global positioning system (GPS) data acquisition.

Initial instrument performance has been verified on the laboratory prototype shown in Figure 2. This prototype provides the identical geometry and ion physics 
as the fieldable unit, while providing the increased flexibility required during the research stage of this program. The results to date have demonstrated that high molecular resolution and sensitivity are achievable with a miniaturized flight tube geometry. Additionally, the system has demonstrated fast response as the entire mass range is acquired in approximately 10 micro seconds. And, for the hand-portable system, where data averaging will be employed, accurate spectra of the sample may be available in less than 1 minute.

The sensor system's unprecedented portability and automation facilitates several unique deployment scenarios. For example, an investigator may easily backpack several of these instruments into the field over rough terrain. Once in position, the high level of automation will enable one operator to perform analyses on multiple systems simultaneously. In addition to verifying the analysis on a second instrument, this scenario will provide for more complete inspections in a limited time frame, and shorter durations in hazardous areas. Additional advantages of this system include automated pattern recognition that can provide the operator near real-time feedback. Pattern analysis can be used to signal the operator of impending danger, suggest additional in-field analysis, and recommend extensive sampling of selected specimens for further laboratory analysis. Enhanced configurations of the system may include a laser desorption sampling system that can photo-ablate molecules and atoms off of solid surfaces by providing a high intensity infra-red laser pulse. Ablated molecules would then be analyzed by leaking into the spectrometer chamber.

In addition to serving the intelligence community in nuclear nonproliferation operations, the sensor system is sufficiently robust to support the in-field sensing requirements of other government and civilian agencies. For example, the system can easily be modified to perform real-time bomb detection applications in airport scanner systems. Other application of this technology will include in-field drug sensing and.identification, and environmental contaminant detection and monitoring.

*We are pleased to acknowledge that the financial support for this work was provided by the U.S. Department of Energy, Office of Intelligence and National Security, Office of Research and Development, under Contract W-31-109-Eng-38. 


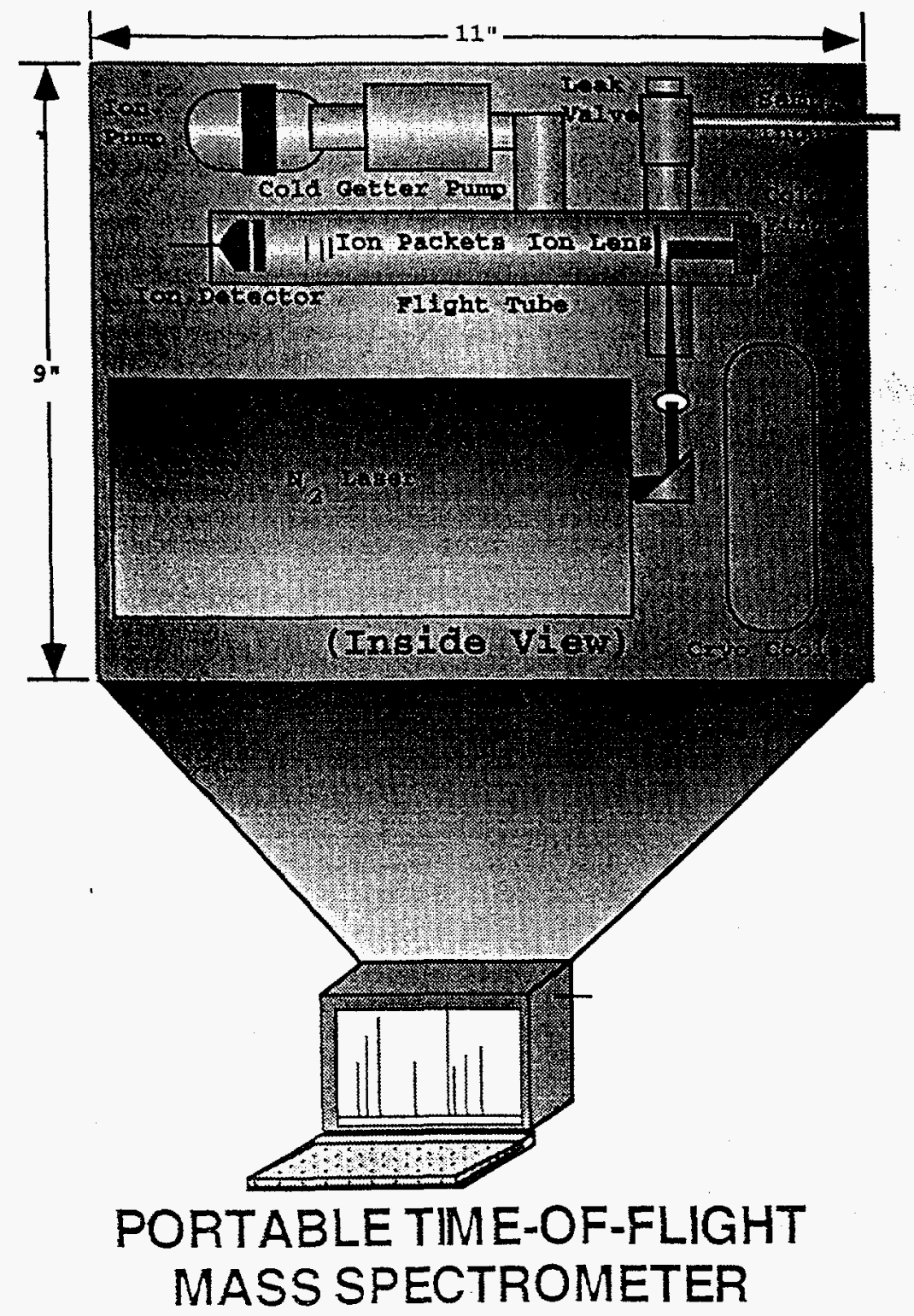

Figure 1. 


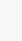

Figure 1. Diagram of the hand-portable sensor system incorporating laser photoionization time-of-flight mass spectrometry. 


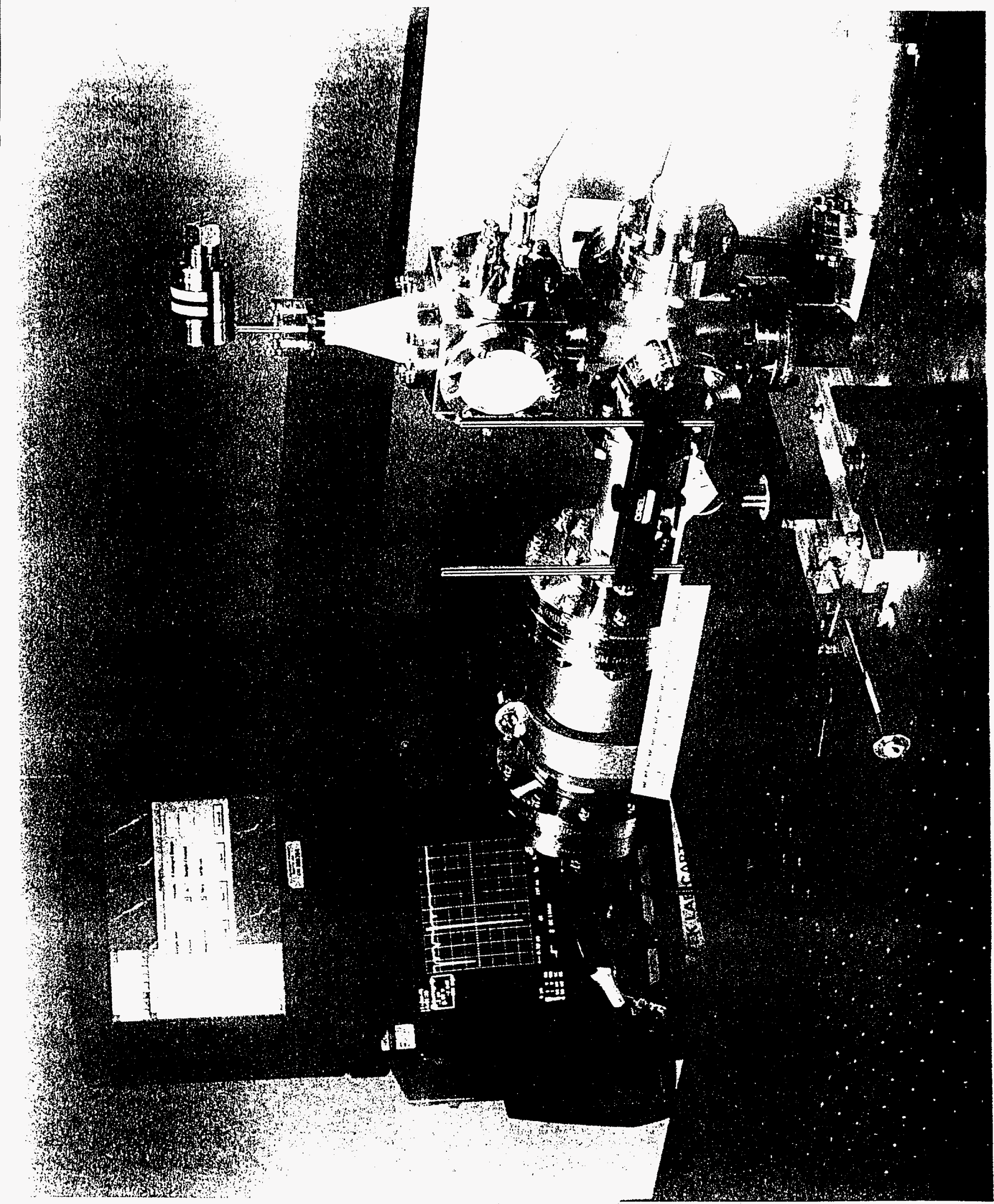




\section{DISCLAMMTER}

Portions of this document may be illegible in electronic image products. Images are produced from the best available original document. 